\title{
Nivel de conocimientos sobre alimentación saludable y grado de aceptabilidad de menús, por estudiantes residentes del Servicio de alimentación de una universidad privada de Lima
}

\author{
Level of knowledge about healthy feeding and grade of acceptability of the menus in \\ the feeding service by the resident students in a private University in Lima, 2016
}

\author{
Zulma Elisa Aguirre Paredes ${ }^{1^{\star}}$, Sonia Ccoto Huallpa ${ }^{1}$
}

\begin{abstract}
RESUMEN
Objetivo: Determinar la relación del nivel de conocimiento sobre alimentación saludable y grado de aceptabilidad de los menús del servicio de alimentación por los estudiantes residentes de una universidad privada de Lima, 2016. Materiales y métodos: Es un estudio de tipo descriptivo, correlacional de corte transversal. La muestra estuvo conformada por 268 estudiantes seleccionados mediante muestreo no probabilístico de tipo intencional. Se diseñó el cuestionario para medir nivel de conocimiento sobre alimentación saludable con una confiabilidad de 0.732 , y el segundo para medir el grado de aceptabilidad de los menús con una confiabilidad de 0.926, aplicado a estudiantes universitarios del servicio de alimentación durante una semana. Resultados: Muestran que no existe relación estadísticamente significativa entre el nivel de conocimiento sobre alimentación saludable y grado de aceptabilidad de los menús ofrecidos por el servicio de alimentación de una universidad privada de Lima $(p=0,300)$. Con respecto al nivel de conocimiento sobre alimentación saludable $53.4 \%$ tiene un nivel regular y solo el $43.3 \%$ tiene un nivel alto. Por otro lado, el $56 \%$ tienen regular grado de aceptabilidad de los menús ofrecidos. Conclusiones: Se concluye que no existe correlación estadísticamente significativa entre el nivel de conocimiento sobre alimentación saludable y el grado de aceptabilidad de los menús del servicio de alimentación.
\end{abstract}

Palabras claves: Nivel de conocimiento, alimentación saludable, aceptabilidad, menú. (Fuente: DeCS BIREME)

\begin{abstract}
Objective: Determine the relationship of the level of knowledge about healthy feeding and grade of acceptability of the menus in the feeding service by the resident students in a private University in Lima, 2016. Materials and Methods: The type of study is descriptive, correlational cross-section. The sample was comprised of 268 students selected by sampling non-probability of intentional type. The questionnaire was designed to measure the level of knowledge about healthy feeding with a reliability of 0.732 , and the second to measure the degree of acceptability of menus with a reliability of 0.926 , applied to university students of the food service during a week. Results: They show that it doesn't exist a significant relationship statistically between the level of knowledge about healthy feeding and degree of acceptability of the menus offered by the feeding service in a private University in Lima $(p=0,300)$. According with the level of knowledge about healthy feeding $53.4 \%$ has a regular level and only $43.3 \%$ a high level. In the other hand, the $56 \%$ has a good degree of acceptability of the offered menus. As a conclusion, it doesn't exist a significant correlation statistically between the level of knowledge about healthy feeding and the degree of acceptability in the menus of the Feeding Service.
\end{abstract}

Keywords: Level of knowledge, healthy feeding, acceptability, menu. (Source: MeSH NLM)

${ }^{1}$ Nutricionista, Servicio de Alimentación, Universidad Peruana Unión, Lima, Perú. 


\section{INTRODUCCIÓN}

La población, en general, tiene desconocimiento en relación a las características de una alimentación correcta. Esta es una de las causas por las que se torna difícil tener un adecuado estado nutricional. Los jóvenes pasan por alto el desayuno, el almuerzo y consumen una gran cantidad de refrigerios, dulces y snacks; además, consumen alimentos con alto contenido de ácidos grasos saturados, insuficiente fibra y bajo contenido de vitaminas y minerales (Samar, 2014). La falta de conocimiento sobre la alimentación saludable contribuye con el aumento de las enfermedades crónicas no transmisibles, esto se debe a la práctica de hábitos alimentarios poco apropiados, que produce una serie de consecuencias que perjudican el estado de salud, e influyen en la calidad de vida de las personas (Neira, 2014).

En los servicios de alimentación colectiva de instituciones públicas y privadas, la alimentación debe de ser óptima con todos los nutrientes necesarios para conseguir un estado nutricional adecuado y que cubra las necesidades nutricionales de los estudiantes (Aparicio \& Avila, 2014).

La OMS (2016), informó que más del 39\% o 1900 millones de adultos de 18 años a más tenían sobrepeso, y $13 \%$ o 600 millones eran obesos. La mayoría de la población mundial vive en países donde el sobrepeso y la obesidad cobran más vidas de personas que están debajo del peso saludable.

En Perú, la tendencia de las curvas de sobrepeso y obesidad han ido en aumento en los últimos 40 años, según la Encuesta de Nutrición del Poblador Peruano (ENNPE 1975 - 2005-2015) (Pajuelo, 2015) con cifras de $24.9 \%, 32.6 \%$ al $34.7 \%$ de sobrepeso respectivamente y del $9 \%$, $14 \%$ al $17.5 \%$ para la obesidad en personas de 15 a más años de edad.

Pozo et al. (2013), evaluó los conocimientos previos en alimentación/nutrición y su relación con el Índice de Masa Corporal (IMC) en una población de estudiantes en España. Para ello, usó una metodología cuantitativa, descriptiva correlacional, utilizando un cuestionario que permite conocer conocimientos previos erróneos sobre alimentación, aplicado a una muestra conformada por 30 alumnos de $2^{\circ}$ de bachillerato. Los resultados más significativos muestran diferencias conceptuales en los contenidos de alimentación y nutrición. Se encontró que las mujeres $(73.5 \%)$, tenían alto grado de conocimiento con respecto a alimentación y nutrición que los hombres (26.5\%, y un nivel de significación del $5 \%(p<0,05)$. Se concluyó que los estudiantes de mayor IMC son los que más conocimientos erróneos presentan, recomendándose una intervención educativa.

En el año 2013, Ecenarro y Dermachi (2013) realizó una investigación, en Argentina. Su objetivo fue evaluar el grado de aceptación de los menús de un nuevo servicio de distribución de alimentos implementados, en niños a partir de 7 años, y adultos que reciben el menú general, y establecer la asociación entre la aceptabilidad y la temperatura del servido de un hospital. El tipo de estudio fue descriptivo, transversal y correlacional. La muestra del estudio fue 380 adultos y 20 niños, para la recolección de datos para la aceptabilidad se usaron un cuestionario con escala hedónica y para la temperatura se usó un termómetro digital profesional. En conclusión, en el cambio de sistema del servicio de alimentación de tradicional a centralizada, las aceptaciones finales de los menús ofrecidos fueron muy satisfactorias. En todas las preparaciones se halló una perdida notoria de temperatura por debajo a la recomendada por el Ministerio de Salud y consenso europeo, las sopas en todas sus versiones no fueron aceptadas, mientras que la carne y la guarnición fueron consumidas en su totalidad. Las variables sabor, variedad, tamaño de porción y tiempo disponible para comer, son las que más intervinieron en el resultado final de la aceptabilidad.

Por lo expuesto, el objetivo del estudio es determinar la relación entre el nivel de conocimiento sobre alimentación saludable y grado de aceptabilidad de los menús ofrecidos a estudiantes residentes por el servicio de alimentación de una universidad privada de Lima.

\section{MATERIALES Y MÉTODOS}

El diseño del estudio es de enfoque cuantitativo, no experimental, de corte transversal, de tipo descriptivo y correlacional. Las variables del estudio fueron nivel de conocimiento sobre alimentación saludable y grado de aceptabilidad de menú. La población del estudio fue de 268 estudiantes residentes de 16 a 29 años de edad 

del Servicio de alimentación de una universidad privada de Lima

de una universidad privada. Los participantes fueron seleccionados mediante el muestreo no probabilístico de tipo intencional, considerando criterios de inclusión y exclusión.

Para la recolección de datos se diseñó un instrumento que consta de tres partes: Fichas sociodemográficas; conformado por los siguientes ítems: género, edad, estado civil, nivel de instrucción, lugar de procedencia, religión, facultad, ciclo de estudio, tiempo de residencia y régimen alimentario. Para los conocimientos aplicó un cuestionario que constó de 14 ítems relacionados al nivel de conocimiento sobre alimentación saludable. El cuestionario tuvo respuestas de opción múltiple. Las respuestas se codificaron en dos opciones: las respuestas falsas con 0 y las respuestas verdaderas con 1 . El valor final fue de la siguiente manera: bueno (11- 14 puntos), regular (5-10 puntos) y malo (0-4 puntos), y fue creado por las investigadoras, validado por juicio de expertos, hallándose una adecuada validez ( $\mathrm{V}$ de Aiken=1), encontrándose una confiabilidad de 0.732 , según la prueba estadística KR20. El cuestionario para aceptabilidad de menús estuvo conformado por 15 ítems sus respuestas fueron categorizadas y codificadas de la siguiente manera: malo $=0$, regular=1 y bueno=2. El valor final fue de la siguiente manera: bueno (21- 30 puntos), regular (11-20 puntos) y malo (0-10 puntos). El instrumento fue elaborado por López, el 2013 en
Guatemala y adaptada para esta investigación, validado por el juicio de expertos, se hizo una prueba piloto con 60 participantes donde se encontró adecuada confiabilidad, con un Alfa de Cronbach de 0.926 .

En primer lugar, se hizo las coordinaciones con la Institución y se solicitó permiso al comité de (DGI) de la Universidad Peruana Unión, para aplicar los cuestionarios a estudiantes residentes de esta institución. Así mismo, se solicitó autorización a la Jefatura del servicio de alimentación. luego, se explicó a los participantes sobre los objetivos de la investigación y finalmente, se leyó el consentimiento informado para la participación voluntaria. Lo datos obtenidos fueron ingresados al programa SPSS en su versión 22 con el propósito de analizar el nivel de conocimiento sobre la alimentación saludable y el grado de aceptabilidad de los menús. Para el análisis descriptivo se utilizó tablas de frecuencia y porcentaje y para determinar la relación entre las dos variables de estudio se utilizó la prueba estadística Chi Cuadrado.

\section{RESULTADOS}

En la Tabla 1, se observa que el $53.4 \%$ tiene conocimiento regular y solo $43.3 \%$ tiene conocimiento alto.

Tabla 1

Distribución de la muestra del nivel de conocimiento sobre alimentación saludable.

\begin{tabular}{lcc} 
Nivel de conocimiento & N & $\%$ \\
Bajo & 9 & 3.4 \\
Regular & 143 & 53.4 \\
Alto & 116 & 43.3 \\
Total & 268 & 100.0 \\
\hline
\end{tabular}

En la Tabla 2, se observa que el $56 \%$ de los estudiantes tienen aceptabilidad regular de los menús ofrecidos por el servicio de alimentación y solo el $28 \%$ aceptabilidad buena. 
Tabla 2

Distribución de la muestra del grado de aceptabilidad de los menús del servicio de alimentación.

\begin{tabular}{lcc} 
Grado de aceptabilidad & N & $\%$ \\
Malo & 43 & 16.0 \\
Regular & 150 & 56.0 \\
Bueno & 75 & 28.0 \\
Total & 268 & 100.0 \\
\hline
\end{tabular}

En la Tabla 3, se observa que no existe relación estadística significativa entre el nivel de conocimientos sobre alimentación saludable y el grado de aceptabilidad de los menús.

Tabla 3

Análisis de relación entre el nivel de conocimiento sobre alimentación saludable y el grado de aceptabilidad de los menús.

\begin{tabular}{|c|c|c|c|c|c|c|}
\hline \multirow{2}{*}{\multicolumn{2}{|c|}{$\begin{array}{l}\text { Nivel de } \\
\text { conocimientos }\end{array}$}} & \multicolumn{3}{|c|}{ Aceptabilidad } & \multirow{3}{*}{$\begin{array}{l}\text { Total } \\
9\end{array}$} & \multirow{3}{*}{$\begin{array}{l}\text { Chi cuadrado } \\
X 2=4.874\end{array}$} \\
\hline & & \multirow{2}{*}{$\begin{array}{l}\text { Malo } \\
3\end{array}$} & \multirow{2}{*}{$\begin{array}{l}\text { Regular } \\
4\end{array}$} & \multirow{2}{*}{$\begin{array}{l}\text { Bueno } \\
2\end{array}$} & & \\
\hline Bajo & $\mathrm{n}$ & & & & & \\
\hline & $\%$ & $1.1 \%$ & $1.5 \%$ & $0.7 \%$ & $3.4 \%$ & $p=.300$ \\
\hline \multirow[t]{2}{*}{ Regular } & $n$ & 25 & 74 & 44 & 143 & \\
\hline & $\%$ & $9.3 \%$ & $27.6 \%$ & $16.4 \%$ & $53.4 \%$ & \\
\hline \multirow[t]{2}{*}{ Alto } & $\mathrm{n}$ & 15 & 72 & 29 & 116 & \\
\hline & $\%$ & $5.6 \%$ & $26.9 \%$ & $10.8 \%$ & $43.3 \%$ & \\
\hline \multirow[t]{2}{*}{ Total } & $\mathrm{n}$ & 43 & 150 & 75 & 268 & \\
\hline & $\%$ & $16.0 \%$ & $56.0 \%$ & $28.0 \%$ & $100.0 \%$ & \\
\hline
\end{tabular}

\section{DISCUSIÓN}

Una alimentación se denomina saludable siempre y cuando aporte todos los nutrientes necesarios para el buen funcionamiento y mantenimiento de todos las funciones vitales del organismo (FAO/ WHO/UNU \& Consultation, 2001). Conocer sobre alimentación saludable ayuda a elegir adecuadamente a fin de prevenir trastornos causados por excesos, déficit o desequilibrios. Las conductas alimentarias están acompañadas de algunos conocimientos previos que el joven posee, adquirido en el hogar, entorno o centro de estudios; es entonces, que surge la necesidad de mejorar esos conocimientos para fomentar hábitos saludables (Mondalgo, 2016).

La aceptabilidad de los menús constituye un factor en la determinación de la calidad que perciben los estudiantes, la presentación de las preparaciones que brinda un servicio de alimentación y las características organolépticas de los menús (Thimoteo, Assunsion, Ribeiro, De Oliveira, \& Stedefeldt, 2013). Esta aceptabilidad es el resultado de la interacción del alimento y el hombre en un determinado momento. Las características del alimento como la composición química, nutricional, estructural y propiedades físicas cumplen un papel importante en el momento de la elección de los alimentos. El entorno de cada consumidor está rodeado de hábitos familiares, religión y educación que influyen en el momento de aceptar o rechazar un alimento (Costell, 2001).

Al evaluar el nivel de conocimiento de los estudiantes residentes, se evidenció que el $53.4 \%$ tiene un nivel regular y solo el $43.3 \%$ un nivel alto. Estos resultados probablemente están relacionados con la edad de los estudiantes siendo que el $(60.7 \%)$ tienen edades entre 1618 años, algunos de ellos tienen conocimientos previos impartidos en el hogar, más no tienen la información completa. Al evaluar las preguntas se encontró que los estudiantes no reconocen qué alimentos aportan energía, tipos de regímenes, funciones de las vitaminas, y creen que las guarniciones, postres y refrescos 
Nivel de conocimientos sobre alimentación saludable y grado de aceptabilidad de menús, por estudiantes residentes del Servicio de alimentación de una universidad privada de Lima

deben formar parte de un menú completo. Similar resultado encontró Vera (2015), donde el $50 \%$ de los estudiantes tenían un nivel de conocimiento medio y el $42 \%$ nivel alto. Por otro lado, Gámbaro et al. (Gámbaro, Raggio, Dauber, Ellis, \& Toribio, 2011) y Delbino (2013) evidenciaron que los estudiantes más del $50 \%$ tuvieron nivel de conocimiento bueno.

Al evaluar el nivel de conocimiento con el sexo de los estudiantes se encontró que las mujeres tienen nivel de conocimiento alto (48.4\%) en comparación con los varones (39\%). Las referencias mencionan que las mujeres son las que constantemente buscan información sobre como verse bien y qué alimentos cumplen esa función. Otra percepción que se tiene es que las mujeres son las que conocen de preparación, de compras, de selección más que los varones. Son ellas las que, al momento de servirse de un menú, preguntan qué ingredientes tiene la preparación, lo que permite que actualice la información previa que recibió del entorno o vida diaria. Pozo et al. (2013) también encontraron que el $(73.5 \%)$ de las mujeres tenían mayor grado de conocimiento con respecto a alimentación y nutrición que el (26.5\%) de los hombres.

Con respecto al régimen alimentario, se halló que del total de 268 estudiantes el $49.3 \%$ son omnívoros y el $25.4 \%$ lacto ovo vegetariano, del total de lacto ovo vegetariano el $52.9 \%$ tienen nivel de conocimiento alto en comparación con los omnívoros, uno de los datos sociodemográficos es la afiliación religiosa, el $88.8 \%$ de los estudiantes son adventistas. Se asume que conocen los remedios naturales, los problemas del consumo de carnes, la importancia de comer más vegetales. Freeland et al.(1982), encontraron que los vegetarianos $(p<0.005)$ obtuvieron puntuaciones significativamente más altas que los omnívoros $(p<0.01)$.

A la evaluación del grado de aceptabilidad del menú, se encontró que el $56 \%$ de los estudiantes tienen regulargrado de aceptabilidad y solo el $28 \%$ tienen un buen grado. Al evaluar las preguntas se encontró que los estudiantes no aceptan las preparaciones de las cenas, porque refieren que es poca cantidad, algunas veces no se puede repetir porque existen algunas preparaciones en porciones individuales; además, refieren que no les gusta cómo se sirven las comidas, se observa que ellos prefieren una atención tipo buffet donde puedan servirse la cantidad y la preparación que desean. Pari y Llihua (2015) demostraron que el $59,7 \%$ de estudiantes tenían un nivel de aceptabilidad media en relación con las características del menú: horario, variedad de menú, temperatura, sabor, composición y cantidad de ración. No obstante Ecenarro y Dermachi (2013) encontraron que el $87.5 \%$ de los estudiantes tenían buena aceptabilidad de los menús y solo el $11.0 \%$ nivel regular.

Referente al resultado de aceptabilidad de los servicios de desayuno, almuerzo y cena, el $(44 \%)$ tiene mayor aceptabilidad del almuerzo en comparación con los otros alimentos. La percepción que se tiene es que los estudiantes del turno tarde al sonido de la campana del mediodía encuentran el almuerzo caliente, variado, las bandejas de ensaladas llenas, y comen en armonía y la atención de los que vienen después de clase es igual. Esta situación, coincide con (López, 2013) que constató una buena aceptabilidad de almuerzo el $45 \%$ respondió que les gusta el almuerzo respecto a las otras comidas. Este estudio es similar al que encontró (Latorre, 2014) donde los estudiantes tenían un $51 \%$ de aceptabilidad regular y $87 \%$ de aceptabilidad buena.

En relación a los componentes del almuerzo se encontró que el $54.4 \%$ de los residentes tuvieron buena aceptabilidad por las ensaladas que comprenden tres ensaladas frescas $y$ una cocida. Esto se da porque los estudiantes tienen buen conocimiento sobre alimentación saludable, la importancia, del consumo de verduras y hortalizas y aprovechar los elementos antioxidantes de estas. De igual manera, Medina et al. (2007), en su investigación observaron que los estudiantes de 12 a 15 años de edad, prefieren ensaladas, siendo los de mayor preferencia: tomate $(96.6 \%)$, col $(77.9 \%)$, lechuga $(75.4 \%)$ y pepino $(72.8 \%)$. Coincidiendo con los componentes de ensaladas del servicio de alimentación del estudio.

El consumo de refrescos alcanzó una aceptabilidad de $51.9 \%$, se sabe que el alimento no se debe ingerir junto con líquidos, la recomendación es beber media hora antes o después de cada comida, pero por cultura en el Perú el menú incluye refresco o alguna bebida, son pocos estudiantes que no llevan vaso para servirse refresco porque están conscientes del buen cuidado de su salud, y la aceptabilidad también se debe a que los refrescos se sirven a voluntad. Zaragoza et al.(2013) encontraron que el consumo de refrescos naturales en 
estudiantes universitarios menores de 25 años de edad tuvieron aceptabilidad regular (15.4\%).

Otro resultado mostró que el $41 \%$ tiene aceptabilidad de la temperatura de las comidas. En su estudio de López (2013), presentó que el $95 \%$ de los encuestados contestaron que si es adecuado la temperatura de los alimentos servidos. Así como (Redondo, 2006) encontró una buena aceptabilidad entre 75$79 \%$, considerando la temperatura de las preparaciones de pastas, arroz y menestras.

Con respecto a la relación del nivel de conocimiento sobre alimentación saludable y grado de aceptabilidad de los menús del servicio de alimentación se encontró que no existe relación estadísticamente significativa ( $p>.05)$. Estos resultados podrían deberse a los conocimientos nutricionales adquiridos durante las diferentes etapas de la vida, los cuales no influyen en la aceptabilidad de los menús, siendo que las personas que conocen sobre nutrición prefieren el consumo de alimentos saludables (Estrella, 2013). No obstante, Wardle et al. (2000) indicaron que el conocimiento influye en el momento de elegir los alimentos saludables y reportaron que a mayor conocimiento hay mayor ingesta de frutas y vegetales. Asimismo, Patterson et al. (1995) señalaron que los conocimientos tienen influencia en la preferencia y selección de alimentos, mas no en la aceptabilidad de los menús.

Declaración de financiamiento y de conflicto de intereses:

El estudio fue financiado por los autores, quienes declaran no tener conflictos de interés.

\section{Correspondencia:}

Zulma Aguirre Paredes. Dirección: Universidad Peruana Unión, Carretera Central Km 19, Ñaña, Lima, Perú.

\section{Correo electrónico zulmaaguirre@upeu.edu.pe}

\section{REFERENCIAS BIBLIOGRÁFICAS}

Aparicio, V., \& Avila, A. (2014). Aporte nutricional de los almuerzos brindados por un concesionario a estudiantes universitarios. Universidad Peruana de Ciencias Aplicadas.

Costell, E. (2001). La aceptabilidad de los alimentos: nutrición y placer. Arbor, (661), 65-85. https://doi. org/10.3989/arbor.2001.i661.823.

Delbino, C. (2013). Conocimientos, actitudes y prácticas alimentarias en adolescentes concurrentes al colegio F.A.S.T.A. Universidad FASTA.

Ecenarro, C., \& Demarchi, J. (2013). Aceptación de menús ofrecidos por un servicio de alimentación con producción centralizada en el hospital J.B. Itarruspe, Santa Fé.

Estrella, C. (2013). Factores relacionados con las prácticas alimentarias en el consumo de alimentos ofertados en el bar scolar de adolescentes de colegio "La presentación."

FAO/WHO/UNU, \& Consultation, E. (2001). Human energy requirements. Rome.

Freeland, J., Greninger, S., Vickers, J., Bradley, C., \& Young, R. (1982). Nutrition knowledge of vegetarians and nonvegetarians. Journal of Nutrition Education, 14(1), 21-26. https://doi. org/10.1016/S0022-3182(82)80058-7

Gámbaro, A., Raggio, L., Dauber, C., Ellis, A., \& Toribio, Z. (2011). conocimientos nutricionales y frecuencia de consumo de alimentos: un estudio de caso. Archivos Latinoamericanos de Nutrición, 61(3), 315-315.

Latorre, P. (2014). Diseño y Optimización de Minutas para Comedores Principales. Escuela Superior Politécnica del Litoral.

López, D. (2013). Determinación de valor nutritivo y aceptación de las dietas servidas a lo pacientes que ingresan al hospital nacional Dr. Moisés Villagran Mazariegos, del departamento de San Juan marcos, Guatemala. (Tesis Maestría). Universidad Rafael Landivar.

Medina, A., Guridi, M., Ramón, F., \& Pérez, M. (2007) Comportamiento del consumo de vegetales en adolescentes de un área de salud. Revista Psicológica Científica.24(3) 20-32

Mondalgo, J. (2016). Efectividad de un programa educativo en el nivel de conocimiento sobre alimentación saludable en los adolescentes de una Institución Educativa . Lima - 2015. (Tesis 
Nivel de conocimientos sobre alimentación saludable y grado de aceptabilidad de menús, por estudiantes residentes del Servicio de alimentación de una universidad privada de Lima

Licenciatura). Universidad Nacional Mayor de San Marcos.

Neira, S. (2014). Caracterización de justificaciones dadas por escolares del Colegio José Max León frente a elecciones alimentarias: un análisis cualicuantitativo. Pontificia Universidad Javeriana.

OMS. (2016). Obesidad y sobrepeso, 1-6.

Pajuelo, J. (2015). El Sobrepeso y La Obesidad En El Perú: Un Problema a Enfrentar.

Pari, L., \& Llihua, K. (2015). Composición de dietas y nivel de satisfacción sobre servicio del comedor de estudiantes, sede central (Tesis Licenciatura). Universidad Nacional de Huancavelica.

Patterson, R., Kristal, A., Lynch, J., \& White, E. (1995). Diet-cancer related beliefs, knowledge, norms, and their relationship to healthful diets. Journal of Nutrition Education, 27(2), 86-92. https://doi. org/10.1016/S0022-3182(12)80348-7

Pozo, A., Cubero, J., \& Ruiz, C. (2013). Conocimientos previos en alimentación y nutrición y su relación con el índice de masa corporal de un grupo de estudiantes de un penitenciario español; un recurso en educación para la salud. Revista de La Facultad de Educación Para Albacete, 28, 87-101.

Redondo, S. (2006). Diseño de un sistema de monitoreo de la calidad del menú y satisfacción al cliente interno del servicio de alimentos del centro nacional de rehabilitación. Universidad de Costa Rica.
Samar, E. (2014). Cómo lograr una vida saludable. Anales Venezolanos de Nutrición, 27(1), 129142.

Thimoteo, D., Assunsion, R., Ribeiro, R., De Oliveira, L., \& Stedefeldt, E. (2013). Métodos para las pruebas de aceptación para la alimentación escolar: validación de la tarjeta lúdica. Revista Chilena de Nutrición, 40(4), 357-363.

Vera, M. (2015). Conocimiento sobre alimentación saludable y la elección de alimentos por parte de los adolescentes dentro del kiosco escolar. Universidad de la Cuenca Del Plata.

Wardle, J., Parmenter, K., \& Waller, J. (2000). Dietary restraint and self-reported meal sizes: diary studies with differentially informed consent. Appetite, 34(3), 269-275. https://doi.org/10.1006/ appe. 2000.0314

Zaragoza, M., Norte, A., Fernandez, J., Sánchez, J., \& Ortiz, R. (2013). Tipo de bebidas consumidas por los estudiantes universitarios. Revista Española de Nutrición Comunitaria, 19(2), 114-119.

Recibido: $14 / 05 / 17$

Aceptado: 18/10/17 